\title{
DOENÇA CEREBROVASCULAR E NEUROCISTICERCOSE
}

\author{
Maria Sheila Guimarães Rocha', Sônia Maria Dozzi Bruck², \\ Ana Cláudia Ferraz ${ }^{3}$ Ana Cláudia Piccolo ${ }^{3}$
}

\begin{abstract}
RESUMO - Apresentamos três casos de arterite do sistema nervoso central associada a neurocisticercose. No primeiro caso, relatamos a ocorrência de arterite bilateral das artérias cerebrais médias em um paciente de 36 anos, com quadro de hemiparesia direita e afasia. A ressonância nuclear do encéfalo evidenciou cisto racemoso parietal direito e infarto temporal esquerdo. 0 estudo angiográfico mostrou oclusão total da artéria cerebral média esquerda e oclusão subtotal da artéria cerebral média direita. No segundo caso demonstramos a ocorrência de vasculite de pequenos vasos em um paciente de 42 anos, com quadro de cefaléia, crises convulsivas, afasia e déficit motor. A tomografia de crânio revelou calcificações intraparenquimatosas e área isquêmica temporal esquerda. 0 estudo angiográfico cerebral revelou-se normal. 0 terceiro caso trata de uma paciente de 53 anos de idade com história pregressa de seis episódios de acidente vascular cerebral e quadro atual de distúrbio do comportamento e convulsões. A tomografia e ressonância nuclear magnética de crânio revelaram múltiplos infartos lacunares e cistos cisternais. A angiografia cerebral mostrou arterite de vasos intracranianos tanto do sistema carotídeo como vértebro-basilar. Nos três casos o estudo quimiocitológico do líquor mostrou pleocitose linfomonocitária e reação imunológica (ELISA) para cisticercose positiva.
\end{abstract}

PALAVRAS-CHAVE: neurocisticercose, acidente vascular cerebral, arterite cerebral.

\section{Cerebrovascular disease and neurocysticercosis}

\begin{abstract}
We report three cases of stroke secondary to neurocysticercosis. The first one is a 36 years old man with bilateral middle cerebral artery occlusions who had presented acute right hemiparesia and aphasia. MRI demonstrated several enhancing subarachnoid cysts surrounding the occluded vessels, a right parietal racemose cyst and a left temporal large infarction area. Angiographic study showed total occlusion of left middle cerebral artery and a subtotal occlusion of right middle cerebral artery. The second one is a 42 years old man with vasculitis of small cortical vessels who presented with headache, seizures and focal neurological deficit. CT scan demonstrated several calcifications and a left temporal infarction area. Cerebral angiographic study was normal. The third case was a woman, 53 years old, with a past history of six stroke events and an actual behavior disturbance and seizures. MRI demonstrated several cortical and subcortical infarction areas and cisternal cysts. Angiographic study showed diffuse arteritis of basilar and carotid arterial system. In all three cases CSF study showed linfomonocitic pleocytosis and positive ELISA for cysticercosis.
\end{abstract}

KEY WORDS: neurocysticercosis, stroke, arteritis.

A cisticercose é a doença parasitária que mais frequentemente atinge o sistema nervoso central (SNC), sendo endêmica em muitos países subdesenvolvidos, especialmente na América Latina, Central e na Áfri$\mathrm{ca}^{1}$. Apresenta frequência crescente em países desenvolvidos em consequência da constante imigração a partir de regiões endêmicas ${ }^{2}$. Com altas taxas de morbidade e de letalidade, a neurocisticercose constitui grave problema de saúde pública, apresentando desastrosos reflexos sócio-econômicos. A neurocisticercose é doença que assume várias formas de apresentação e evolução clínica, sendo uma de suas complicações a arterite cerebral ${ }^{3,4}$. Pouco reconhecida até muito recentemente, a arterite cerebral secundária à neurocisticercose foi relatada em poucas publicações científicas ${ }^{5-8}$. Não encontramos publicação anterior em âmbito nacional, apesar da alta taxa de prevalência da neurocisticercose em nosso meio.

A doença vascular cerebral ocasionada pela neurocisticercose pode ocorrer espontaneamente ou na vigência do tratamento anti-parasitário, sendo também descrita como primeira manifestação clínica da

Serviço de Neurologia Clínica do Hospital Santa Marcelina, São Paulo SP, Brasil (SN-HSM): 'Doutora em Medicina, Chefe do SN-HSM; 2Doutora em Medicina; ${ }^{3}$ Mestre em Neurologia, Médica Assistente do SN-HSM.

Recebido 12 Março 2001, recebido na forma final 29 Maio 2001. Aceito 7 Junho 2001. 
doença ${ }^{8}$. Apesar do aumento do número de relatos de pacientes com infartos cerebrais devidos à neurocisticercose, esta situação clínica persiste subestimada como diagnóstico diferencial das doenças cerebrovasculares.

Apresentamos neste artigo três casos de doença vascular cerebral decorrente da infestação cisticercótica do SNC. Nos três casos relatados os eventos isquêmicos cerebrais não estavam associados ao tratamento parasiticida.

\section{CASOS}

Caso 1. Trata-se de homem, de 36 anos de idade, internado no Serviço de Neurologia (SN) do Hospital Santa Marcelina (HSM) com quadro de hemiparesia direita e afasia. Há aproximadamente quatro anos o paciente vinha apresentando quadro de crises convulsivas tônico-clônicas generalizadas e em duas situações no último ano apresentou déficit motor transitório no dimídio esquerdo. A ressonância nuclear do encéfalo (RM) evidenciou cisto racemoso parietal direito e grande área de infarto temporal esquerdo (Fig 1-A e C). $O$ estudo angiográfico mostrou oclusão total da artéria cerebral média esquerda e oclusão subtotal (95\%) da artéria cerebral média direita (Fig 1-B e D). $O$ estudo quimiocitológico do líquor (LCR) evidenciou pleocitose com predomínio linfomonocitário (54 células:
$90 \%$ linfomonocitárias e $10 \%$ polimorfonucleares), hiperproteinorraquia $(120 \mathrm{mg} / \mathrm{dl})$ e reação imunológica (ELISA) para cisticerco positiva. O paciente foi submetido a procedimento neurocirúrgico para ressecção do cisto racemoso, cujo estudo anatomopatológico confirmou tratarse de cisticerco com necrose e calcificação. Foram realizados exames laboratoriais com o intuito de descartar outras causas de acidente vascular cerebral (AVC), como as vasculites secundárias às doenças do colágeno, outras doenças infecciosas (sífilis e SIDA), doenças hematológicas e as cardiopatias mais frequentes. $O$ paciente não apresentava outros fatores de risco para doença vascular cerebral, tais como: hipertensão arterial sistêmica, cardiopatia, diabetes mellitus, coronariopatia, tabagismo, etilismo ou uso de drogas ilícitas. Tais fatores de risco não foram nem relatados na história clínica nem detectados no exame clínico do paciente. O paciente recebeu tratamento com corticosteróide, evoluiu com melhora do nível de consciência e pequena melhora do déficit motor e da afasia. $\mathrm{O}$ paciente permanece em acompanhamento ambulatorial no SN-HSM.

Caso 2. Homem de 42 anos, que em 1995 apresentou quadro de cefaléia de moderada intensidade, halocraniana e contínua e crises convulsivas do tipo parcial simples motor no dimídio direito com generalização secundária. A tomografia de crânio (TC) nesta ocasião revelou cistos race-

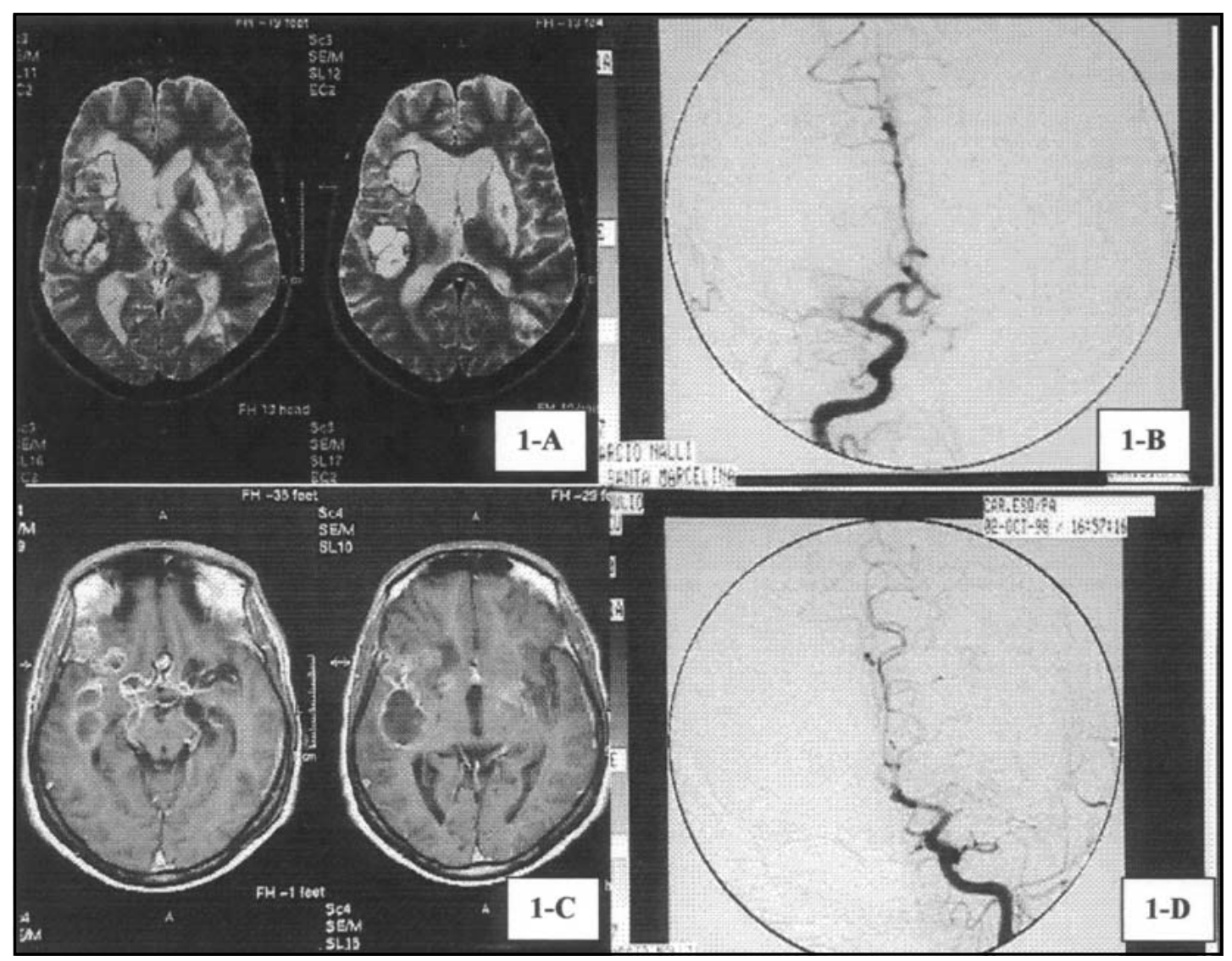

Fig 1. Exames de imagem - ressonância nuclear e angiografia cerebral do paciente do Caso 1. 


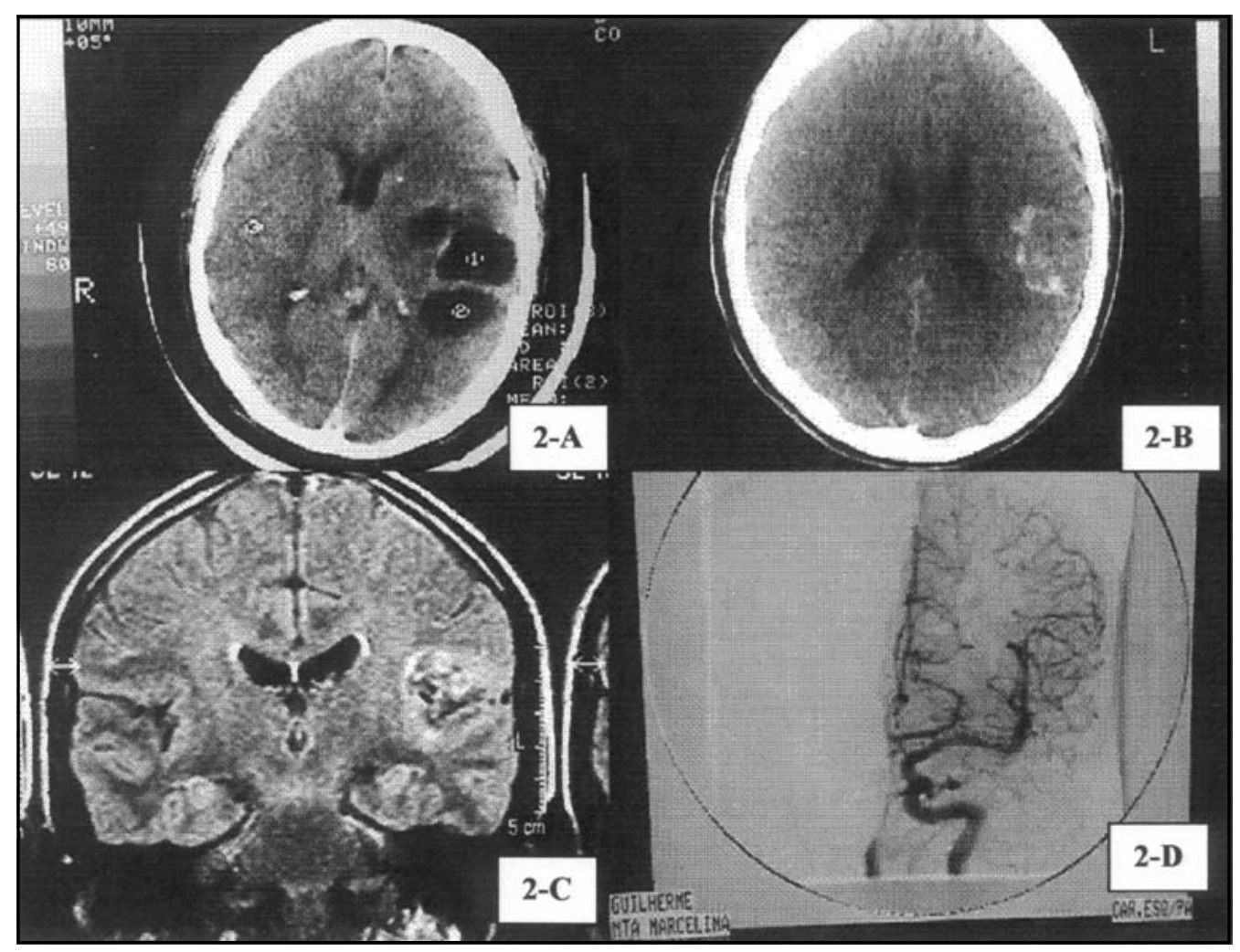

Fig 2. Exames de imagem - tomografia computadorizada, ressonância nuclear e angiografia cerebral do paciente do Caso 2.

mosos no lobo temporal esquerdo e vários cistos viáveis (Fig 2-A). O exame do LCR evidenciou pleocitose com predomínio linfomonocitário (36 células: 98\% linfomonocitárias e $2 \%$ polimorfonucleares), hiperproteinorraquia (95 $\mathrm{mg} / \mathrm{dl}$ ) e reação imunológica (ELISA) positiva para cisticercose. Recebeu tratamento com praziquantel na dosagem de $50 \mathrm{mg} / \mathrm{Kg}$ durante 15 dias associado ao uso de dexametasona na dosagem de $8 \mathrm{mg} / \mathrm{dia}$. O paciente evoluiu com melhora da cefaléia e controle clínico das crises convulsivas, com uso regular de fenitoína $300 \mathrm{mg} / \mathrm{dia}$. Cinco anos após o tratamento da neurocisticercose, o paciente apresentou quadro de hemiparesia direita e afasia de caráter transitório. A TC mostrava pequena área de sangramento irregular na região têmporo-parietal esquerda, interpretada como possível mal-formação artério-venosa (Fig 2-B). A RM revelou área de infarto temporal esquerdo permeada por pequeno sangramento intralesional (Fig 2-C). O estudo quimiocitológico do LCR evidenciou pleocitose linfomonocitária (20 células: 95\% linfomonocitárias e $5 \%$ polimorfonucleares) e hiperproteinorraquia (72 mg/dl). A angiografia cerebral foi considerada normal (Fig 2-D). O quadro foi abordado como vasculite e tratado com corticosteróide, sendo utilizada a prednisona na dosagem de 40 $\mathrm{mg} /$ dia durante dois meses e redução posterior da dose até a completa retirada da droga. O paciente evoluiu bem e com recuperação do déficit motor. Foram realizados exames laboratoriais com o objetivo de descartar outras causas possíveis de vasculite do SNC: hemograma e bioquímica básica do sangue, lipidograma, hormônios tiroidia- nos, mucoproteínas, proteína $C$ reativa, perfil de anticorpos anti-núcleo, pesquisa de células LE, fator reumatóide, dosagem de complemento, anticorpo anticardiolipina, eletroforese de hemoglobina, sorologias para vírus HIV (ELISA) e para sífilis (VDRL e TPHA), e ecocardiograma trans-torácico, sendo todos com resultados normais ou negativo. $\mathrm{O}$ paciente não apresentava outros fatores de risco para doença cerebrovascular, como os já citados no caso anterior.

Caso 3. Trata-se de mulher com 53 anos de idade, procedente da Bahia, internada no SN-HSM com quadro clínico de comportamento inadequado e crises convulsivas, que pela descrição dos familiares pareciam ser do tipo generalizado. Os familiares referiam que após o sexto episódio de AVC, tendo o primeiro ocorrido há oito anos, a paciente tornou-se apática, parou de falar, perdeu o controle esfincteriano e passou a apresentar comportamento agressivo e perda de crítica. Os familiares negavam a existência de qualquer doença anterior que pudesse constituir fator de risco para AVC. Não havia referência a hábitos como tabagismo, etilismo ou uso de drogas ilícitas. A bioquímica básica sanguínea, incluindo a dosagens de íons, função hepática, perfil lipêmico e dosagem de hormônios tiroidianos, achava-se normal. A sorologia para sífilis (VDRL e TPHA) foi negativa. As provas de atividade inflamatória (VHS, mucoproteínas, proteína $C$ reativa) apresentavam todos valores normais, assim como foram negativas a pesquisa de células $L E$, o perfil de anticorpos anti-núcleo e o fator reumatóide. A RM revelou múltiplas áreas de in- 


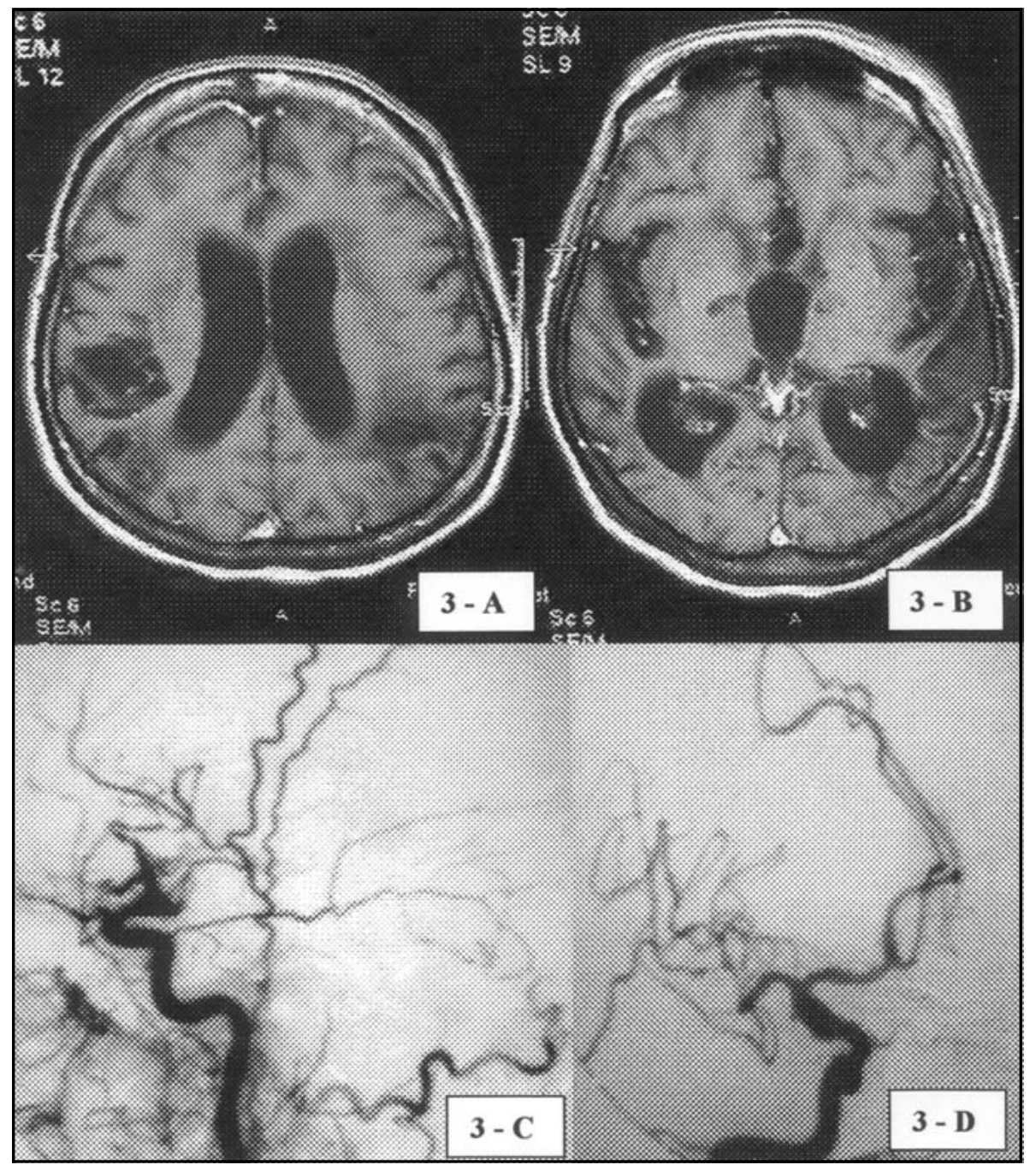

Fig 3. Exames de imagem - ressonância nuclear e angiografia cerebral da paciente do Caso 3. farto em substância branca peri-ventricular, centro semioval, região cápsulo-lenticular bilateral e tronco encefálico, redução volumétrica encefálica e dilatação "ex vacuo" do sistema ventricular cerebral, além de alguns cistos cisternais (Fig 3-A e B). O estudo angiográfico cerebral evidenciou alterações compatíveis com arterite de vários vasos intracranianos, tanto do sistema carotídeo bilateralmente como do sistema vértebro-basilar (Fig 3-C e D). O estudo quimiocitológico do LCR mostrou discreta pleocitose linfomonocitária (18 células: 98\% linfomonocitárias), hiperproteinorraquia (120 mg/dl) com aumento de gamaglobulina com padrão policlonal e reação imunológica (ELISA) positiva para cisticercose. A paciente não recebeu tratamento parasiticida, sendo utilizado a dexametasona na dosagem de $8 \mathrm{mg} / \mathrm{dia}$. A paciente apresentou pouca meIhora do quadro neurológico na evolução clínica.

\section{DISCUSSÃO}

A associação entre cisticercose cerebral e AVC tem sido objeto de discussão na literatura mundial. A frequência de infarto cerebral relacionada a esta parasitose não é bem conhecida, variando entre 2 e
$12 \%$, de acordo com os estudos publicados até este momento ${ }^{4-6,8,9}$. De acordo com grandes séries publicadas, nas quais a associação de neurocisticercose e infarto cerebral foi confirmada, cerca de $3 \%$ dos pacientes com neurocisticercose desenvolvem doença cerebrovascular isqüêmica ${ }^{2}$ e cerca de $2,5 \%$ dos pacientes com infarto cerebral têm neurocisticer$\operatorname{cose}^{9}$. Barinagarrementeira \& Cantú relatam que numa série de 1000 casos de AVC bem documentados, apenas 20 estavam comprovadamente associados à neurocisticercose ${ }^{9}$. Obviamente a proporção de pacientes com doença vascular cerebral devida a neurocisticercose deve variar conforme a prevalência desta parasitose na população à qual pertencem. No México, por exemplo, onde a cisticercose é endêmica, a cisticercose é a segunda causa mais frequente de infarto cerebral de etiologia não-aterosclerótica ${ }^{10}$. No Brasil, onde a cisticercose também é considerada endêmica, não dispomos de dados epidemiológicos publicados em revistas indexadas sobre o assunto.

Da mesma forma, ainda não está definida a fre- 
quência de arterite cerebral, sintomática ou não, nos pacientes com neurocisticercose. São escassos os estudos publicados com documentação angiográfica de arterite cerebral secundária à cisticercose ${ }^{11-15}$. Em estudo recente, os autores Barinagarrementeira \& Cantú ${ }^{14}$ realizaram angiografia cerebral em 28 pacientes portadores de neurocisticercose e observaram alterações angiográficas compatíveis com arterite cerebral em $53 \%$ dos casos, dos quais $80 \%$ apresentavam síndrome clínica neurovascular. Este estudo demonstra ainda que $15 \%$ dos pacientes sem alterações angiográficas apresentavam infartos cerebrais, provavelmente devido ao comprometimento de pequenos vasos. Neste estudo, as artérias mais frequentemente comprometidas foram a cerebral média e a cerebral posterior.

Vários mecanismos parecem estar envolvidos na gênese dos eventos vasculares nos pacientes portadores de neurocisticercose. O comprometimento vascular pode ocorrer tanto nos pequenos vasos da base do cérebro como pode atingir as artérias de calibre médio, este último considerado como mais raro ${ }^{3}$. 0 comprometimento de pequenos vasos está associado ao processo inflamatório presente na base do crânio em vigência de meningite cisticercótica crônica ou recorrente, responsável pelo adelgaçamento das meninges e pela endarterite dos vasos sanguíneos do círculo de Willis ${ }^{3,4,8,16}$. Este tipo de comprometimento é considerado como o responsável pela síndrome vascular lacunar, considerado em alguns estudos como a complicação cerebrovascular mais comum da neurocisticercose $\mathrm{e}^{3-5,17}$. Por outro lado, o comprometimento de vasos de calibre médio produz infartos maiores. A lesão destes vasos pode estar relacionada ao exsudato basal, mas frequentemente associa-se à presença de cistos cisticercóticos localizados na aracnóide que, em contato com as paredes dos vasos, desencadeariam intensa reação inflamatória vascular endotelial levando à fragilidade, deformação e oclusão do vaso. O mesmo mecanismo poderia estar envolvido no desenvolvimento de formação aneurismática, descrita em um único caso na literatura, onde o autor considera que o aneurisma de artéria cerebelar anterior inferior apresentado pelo paciente teria relação com a intensa aracnoidite cisticercótica adjacente ao aneurisma ${ }^{18}$.

Apesar de pouco descrita na literatura científi$\mathrm{ca}^{6,13,15,19,20}$, e considerada como forma rara de complicação neurovascular da cisticercose cerebral ${ }^{3}$, a arterite de vasos de calibre médio foi, no entanto, relatada em alguns estudos angiográficos como sendo o tipo de arterite mais frequentemente observa- do neste tipo de investigação $0^{8,14}$. Provavelmente este paradoxo existe em virtude da dificuldade de se determinar no estudo angiográfico o comprometimento de pequenos vasos. Não podemos deixar de considerar a possibilidade de que este tipo de comprometimento cerebrovascular seja realmente mais freqüente do que fomos capazes de diagnosticar até este momento.

Os casos clínicos apresentados neste estudo constituem variadas formas de apresentação clínica e angiográfica da doença vascular isquêmica cerebral associada a neurocisticercose. Constatamos infartos maiores nos dois primeiros casos, sendo comprovada a arterite de vasos de calibre médio no primeiro e terceiro casos. $O$ estudo angiográfico normal no segundo caso leva a deduzir o comprometimento de vasos menores e corticais. No terceiro caso observamos o comprometimento tanto de vasos pequenos, considerando o padrão lacunar dos infartos observados na RM, como de vasos maiores, comprovado pelo estudo angiográfico.

Nenhum dos pacientes aqui apresentados estava em vigência de tratamento anti-parasitário por ocasião do infarto cerebral, o que descarta a possibilidade de processo inflamatório arterial desencadeado pela droga, cuja ação parasiticida responsável pela destruição dos cistos poderia aumentar a reação inflamatória local. Os pacientes referidos não possuíam outros fatores de risco para doença cerebrovascular. Também foram descartadas outras causas de arterite do sistema nervoso central que pudessem ocasionar os acidentes vasculares cerebrais isquêmicos relatados. Desta forma, parece evidente a relação causal entre a neurocisticercose diagnosticada e os infartos cerebrais desenvolvidos por estes pacientes.

A descrição dos casos acima vem reforçar a observação de outros autores que consideram que a neurocisticercose é uma das causas de $\operatorname{AVC}^{3-5,14,16}$, principalmente nos países onde a cisticercose cerebral é endêmica. $O$ diagnóstico do infarto cerebral secundário a neurocisticercose deve ser estabelecido criteriosamente, considerando o pleomorfismo da própria doença. Devemos ser cautelosos nos casos em que a neurocisticercose e o infarto cerebral possam representar apenas uma associação fortuita, especialmente nos pacientes com idade acima de 45 anos e com outros fatores de riscos para doença cerebrovascular. A relação causal entre a neurocisticercose e o AVC deve estar amparada na evidência tomográfica, ou de RM, de isquemia associada aos achados de imagem sugestivos da cisticercose, 
como calcificações e cistos intraparenquimatosas ou cistos subaracnóideos, e achados liquóricos compatíveis com a aracnoidite ativa por neurocisticercose. É necessário o diagnóstico diferencial com as diversas causas de arterite cerebral.

Em conclusão, o comprometimento vascular cerebral secundário à neurocisticercose deve ser considerado no diagnóstico diferencial etiológico do acidente vascular cerebral isquêmico, principalmente nos pacientes mais jovens e habitantes de áreas endêmicas para a neurocisticercose, sendo necessário que o diagnóstico desta condição seja realizado rápida e apuradamente para que o tratamento precoce possa prevenir a recorrência dos eventos vasculares.

\section{REFERÊNCIAS}

1. Centers for Disease Control and Prevention. Neurocysticercosis update: International Task Force for Disease Erradication. MMWR Morb Mortal Wkly Rep 1992;41:697-698.

2. Scharf D. Neurocysticercosis: two hundred thirty-eight cases from a California hospital. Arch Neurol 1988; 45:777-780.

3. Del Brutto OH. Cysticercosis and cerebrovascular disease: a review. J Neurol Neurosurg Psychiatry 1992;55:252-254.

4. Alarcón F, Hidalgo F, Moncayo J, Vinan I. Cerebral cysticercosis and stroke. Stroke 1992;23:1180-1181.

5. Barinagarrementeira F, Del Brutto $\mathrm{OH}$. Lacunar syndrome due to neurocysticercosis. Arch Neurol 1989;46:415-417.
6. Terpenning B, Litchman CD, Heier L. Bilateral middle cerebral artery occlusions in neurocysticercosis. Stroke 1992;23:280-283.

7. Monteiro L, Almeida-Pinto J, Leite I, Xavier J, Correia M. Cerebral cysticercus arteritis: five angiographic cases. Cerebrovasc Dis 1994;4: 125-133.

8. Cantú C, Barinagarrementeria F. Cerebrovascular complications of neurocysticercosis. Arch Neurol 1996;53:233-239.

9. Barinagarrementeria F, Cantú C. Neurocysticercosis as a cause of stroke. Stroke 1992;23:1180-1181.

10. Barinagarrementeira F. Causas non-aterosclerosas de isquemia cerebral. Arch Inst Nal Neurol Neurocir (Mex) 1989;33.

11. Lombardo L, Mateos H. Cerebral cysticercosis in Mexico. Neurology 1961;11:824-828.

12. Rocca E, Monteagudo E. An angiographic study of neurocysticercosis. Int J Surg 1966;46:130-141.

13. Levy SA, Lillehei KO, Rubinstein D, Stears JC. Subarachnoid cysticercosis with occlusion of the major intracranial arteries: case report. Neurosurgery 1995;36:183-188.

14. Barinagarrementeria F, Cantú C. Frequency of cerebral arteritis in subarachnoid cysticercosis. An angiographic study. Stroke 1998;29:123-125.

15. Kohli A, Gupta R, Kishore J. Anterior cerebral artery territory infarction in neurocysticercosis: evalution by MR angiography and in vivo proton MR spectroscopy. Pediatr Neurosurg 1997;26:93-96.

16. Sangla S, De Broucker T, Abgrall S, Gauthier N. Cerebral infarction disclosing neurocisticercosis. Rev Neurol 1995;151:277-289.

17. Del Brutto OH, Sotelo J. Neurocysticercosis: an update. Rev Infect Dis 1988;10:1075-87.

18. Soto-Hernandez JL, Gomez-Llata Andrade S, Echeverri LA, Teixeira F, Romero V.Subarachonoid hemorrhage secondary to a ruptured inflammatory aneurysm: a possible manifestation of neurocysticercosis: case report. Neurosurgery 1996;38:197-199.

19. McCornick GF, Giannotta S, Zee CS, Fisher M. Carotid occlusion in cysticercosis. Neurology 1983;33:1078-1080.

20. Rodriguez-Cab ajal J, Del Brutto OH, Penagos P, Huebe J, Escobar A. Occlusion of the middle cerebral artery due to cysticercotic angiitis. Stroke 1989;20:1095-1098. 